\title{
Evaluación del programa preventivo DARE en Suecia*
}

\section{LINDSTRÖM, P., SVENSSON, R.}

\author{
National Council for Crime Prevention.
}

Enviar correspondencia a:

PETER LINDSTRÖM. National Council for Crime Prevention. POB 1386. S-11193 Stockholm (Sweden). e-mail: peter.lindstrom@brottsforebygganderadet.se

\section{Resumen:}

La policía sueca ha puesto en marcha un programa educativo de resistencia al abuso de drogas destinado a los alumnos de $7^{\circ}, 8^{\circ}$ y $9^{\circ}$, siendo los propios agentes quienes se encargan de ponerlo en práctica dentro de las aulas. Los autores de este estudio han analizado los efectos del programa VÄGA, una versión del DARE (Drug Abuse Resistance Education) americano. Alrededor de 1.800 alumnos suecos de 22 centros de enseñanza secundaria respondieron en tres ocasiones diferentes, de forma anónima, a una serie de preguntas relacionadas con sus actitudes y experiencias con las drogas.

La curiosidad y la accesibilidad que perciben los jóvenes de esas edades en relación a las drogas ilegales tienen unos efectos estadísticamente significativos sobre las actitudes y las experiencias individuales de los alumnos con dichas sustancias. $\mathrm{Ni}$ las actitudes hacia las drogas ni las experiencias con las mismas de los que han participado en el programa DARE un año antes difieren de las de los jóvenes de su edad que no habían tomado parte en el programa. Finalizamos el estudio con una breve discusión sobre qué medidas debería tomar la policía para impedir la accesibilidad a las drogas y cuál debería ser su papel en las escuelas.

Palabras clave: prevención escolar, policía, evaluación programa, Suecia, actitudes estudiantes

\section{SUMMARY:}

The aim of this study was to analyse the impact student's demand for and perceived availability of illicit drugs have on their attitudes and experience with drugs and also to investigate to what extent a specific police-led school-based drug prevention programme, the project DARE (Drug Abuse Resistance Education) affects student's attitudes and experiences regarding drugs.

As a part in an ongoing evaluation about 1.800 students in 22 Swedish junior high schools on three occasions anonymously answered questions about their attitudes towards and experiences with drugs. The results show that student's curiosity and perceived availability of illicit drugs at the school-level have statistically significant effects on drug-related attitudes and experiences. The attitudes towards and experiences with drugs in the eighth grade of students who participated in the DARE programme in the seventh grade were not different from those students who did not participate in the programme. A brief discussion of what measures the police should conduct in order to block the availability of drugs and what their role in schools should be are finally presented.

Key words: illicit drugs, demand, supply, attitudes, DARE, school programme, evaluation, police, Sweden.

\section{INTRODUCCIÓN}

E principal objetivo de la política seguida en el campo de la droga tanto a nivel nacional como internacional es reducir al máximo el número de individuos que consumen drogas ilegales. Los ciudadanos en general y los jóvenes en particular son animados a mantenerse lejos de dicho consumo a través de unas leyes antidroga y de unas campañas destinadas a influenciar en la opinión pública e informar a la gente de sus efectos nocivos. En Suecia, en la actualidad, se promocionan aquellas medidas que al mismo tiempo restringen la accesibilidad y reducen la deman- da de drogas ilegales. Un informe del Instituto Nacional de Salud Pública para la prevención del consumo del alcohol y otras drogas (Folhälsoinstitutet, 1995) afirma lo siguiente:

"Una estrategia preventiva a largo plazo no hace la menor distinción entre las medidas para restringir la accesibilidad y las medidas para frenar la demanda. Los esfuerzos de la policía por acabar con el tráfico de drogas en las calles están principalmente destinados a restringir la accesibilidad... pero también tienen un efecto sobre la demanda, ya que el fuerte mensaje antidroga de la policía disuade a muchos jóvenes de probar dichas sustancias"

\footnotetext{
* Publicado en Nordisk Alkohol \& Narkotikatidskrift, Vol 15 / 1998 (english supplement)
} 
Aunque no sea necesario hacer una distinción entre las "medidas para restringir la accesibilidad y las medidas para frenar la demanda", es evidente que hay acciones destinadas específicamente a uno u otro tema. La prevención en las escuelas, por ejemplo, tiene como principal objetivo influenciar en la demanda de drogas entre los escolares.

Las cifras publicadas por el Consejo Sueco para la Información sobre el Alcohol y otras Drogas (Centralförbundet för alkohol och narkotikaupplysing, CAN) indican que casi tres de cada cuatro estudiantes de $9^{\circ}$ han realizado algún curso preventivo contra el consumo de drogas, incluyendo alcohol y tabaco, en su centro de enseñanza; de ahí la abreviatura sueca de estos programas educativos "ANT" (alcohol, narcóticos y tabaco). La proporción correspondiente en $6^{\circ}$ es inferior al 70\%. A principios de los ochenta casi el $90 \%$ de los alumnos matriculados entre $6^{\circ}$ y $9^{\circ}$ afirmaban haber participado en un programa educativo ANT (Andersson \& Hibell, 1995-1996). Hoy en día existen numerosos centros escolares en Suecia que comienzan a dar información preventiva a partir de $4^{\circ}$, pero en el manual para la enseñanza de la ley y la justicia de la policía (1995) se incluye un capítulo sobre el tabaco, el alcohol y los narcóticos destinado a niños de $2^{\circ}$. La finalidad es "hacer comprender a los alumnos que siempre deberían decir NO y jamás probar el tabaco, el alcohol o los narcóticos".

Las expectativas de los distintos programas preventivos realizados actualmente en los colegios suecos son muy elevadas. El común denominador de todos ellos es su deseo de cambiar las actitudes de los jóvenes hacia las drogas. Nuestra intención en este artículo es analizar el impacto del mayor programa de prevención contra la droga efectuado en Suecia (el programa VÄGA, el equivalente sueco del programa DARE) sobre las actitudes de los escolares ante la droga y sus experiencias en dicho campo. Asimismo, observaremos de forma empírica cómo la demanda y la accesibilidad influyen en las actitudes de los jóvenes ante las drogas.

El análisis de los efectos de estos dos factores resultarán de sumo interés para poder formular una estrategia preventiva realmente eficaz. Si la accesibilidad resulta tener mayores efectos que la demanda, será necesario tomar medidas que dificulten más el acceso de los jóvenes a las drogas. No se trata únicamente de un asunto en el que deban intervenir la policía, aduanas y otras autoridades dentro del sistema legal vigente; los profesores, comerciantes, padres y demás ciudadanos en general se hallan también en condiciones de poner trabas al fácil acceso a las drogas (Weisburd \& Green, 1995; Green, 1996; Davis \& Lurigio, 1996). Si, por el contrario, la demanda resulta tener mayor influencia sobre el consumo, será necesario tomar medidas que pongan el acento en su reducción. En esta situación, tendremos que ser capa- ces de identificar los programas que mejor se ajusten a dicho objetivo y que resulten más efectivos.

\section{EDUCACIÓN ANT E HIPÓTESIS DE VACUNACIÓN}

Parece estar muy extendida la idea de que cuanto antes y con mayor frecuencia oigan los escolares hablar de los efectos nocivos de las drogas, menos inclinados se sentirán a consumirlas. Todavía nos faltan datos para explicar adecuadamente la asociación entre la educación preventiva y los hábitos de consumo de los jóvenes en Suecia, pero estudios realizados en otros países (por ejemplo, Estados Unidos) indican que el impacto de esta clase de educación sobre la actitud ante las drogas no es en absoluto tan simple ni tan directo. Esto puede aplicarse al programa DARE, modelo del proyecto $\vee \ddot{A G A}$ sueco (para una discusión detallada de las evaluaciones americanas del programa ver Lindström \& Pauloff, 1997).

Los estudios dirigidos por el CAN en los colegios suecos ponen de manifiesto que existe una correlación positiva a largo plazo entre la educación preventiva (ANT) y el porcentaje de alumnos que han probado las drogas; cuanto mayor es el número de estudiantes que han seguido el programa ANT, mayor es el número de jóvenes que han probado las drogas. Es poco probable que ello sea debido a un mecanismo fortuito; resulta mucho más verosímil pensar que las escuelas recurren a los programas educativos ANT cuando perciben que sus estudiantes tienen problemas con las drogas. Sin embargo, incluso cuando un porcentaje mayor de alumnos de $6^{\circ}$ siguen el programa ANT, no existe la menor evidencia de que esto les incline a rechazar posteriormente su consumo. Los estudios que señalan la influencia positiva de los programas de educación preventiva admiten que sus beneficios son comparativamente menores y su duración más corta, y que ello está relacionado sobre todo con las actitudes de los escolares hacia la droga más que con su consumo (reciente investigación de Gottfredson, 1997, y Orlandi, 1996). El Ilamado "Life Skills Training Programme" (LST), dirigido por los profesores o los alumnos de mayor edad, y destinado a los estudiantes de $7^{\circ}$, ha sido positivamente analizado en varias evaluaciones pormenorizadas (Botvin, 1996).

Un estudio sobre los programas educativos de prevención de drogas entre alumnos de $7^{\circ}$ y $9^{\circ}$ de la región de Estocolmo ha llegado a la conclusión de que la profesión externa más involucrada en los mismos es la policía (Lindström, 1997). En más de la mitad de los colegios han invitado a oficiales de policía para dirigirse a los alumnos de $7^{\circ}$. Hace aproximadamente dos años, la policía puso en marcha su propio proyecto para la prevención de drogas, basándose en el modelo DARE americano (Drug Abuse Resistance Education). 
El programa VÄGA (como se conoce en Suecia) consiste en 17 lecciones; la policía se encarga de impartir la mitad de ellas.

El objetivo del programa VÄGA es informar a los estudiantes sobre los tipos más comunes de drogas y sus efectos nocivos, así como "hacerles comprender las graves consecuencias del consumo abusivo de drogas..." (VÄGA: Lärarhandledning 1993). Otra de sus finalidades es que los jóvenes sean más conscientes de la influencia de los amigos y de los medios de comunicación en la elección de una droga. Hay conferencias, ejercicios y juegos en los que todos participan; a través de ellos los estudiantes aprenden distintas técnicas de resistencia que les ayuden a decir NO a las drogas.

En tan sólo cuatro años, el programa VÄGA ha sido llevado a más de 200 colegios de Suecia. Fue seguido por un total de 16.500 alumnos durante el curso escolar 1996/1997. En Estados Unidos, donde el DARE comenzó en 1983, un $70 \%$ de los niños matriculados en los cursos intermedios participan en dicho programa. Hoy en día, es el programa de resistencia a la droga más importante del mundo y se utiliza en unos 40 países (Clayton \& colaboradores, 1996).

\section{INVESTIGACIONES ANTERIORES SOBRE EL PAPEL DE LA ACCESIBILIDADY DE LA DEMANDA}

Las actitudes y experiencias de los jóvenes ante la droga han cambiado en los últimos años. Según las encuestas realizadas anualmente por el CAN entre los colegios del país, el 8\% de los chicos y el 7\% de las chicas de $9^{\circ}$ afirmaron haber consumido drogas en alguna ocasión en 1997. Diez años antes, el porcentaje apenas alcanzaba el 3\%. Las tendencias más positivas de los años ochenta, que redujeron a la mitad el número de jóvenes de 16 años que habían tenido experiencias con las drogas, se han invertido en los noventa. Algo parecido ocurre en muchos otros países, incluyendo Noruega y Estados Unidos (Skretting, 1996; Departamento de Salud y Servicios Humanos de Estados Unidos, 1995).

La curiosidad por las drogas está también aumentando entre los jóvenes escolares. En 1996, la cifra de estudiantes que manifestaron su deseo de probarlas fue la más elevada desde 1971, año en que se tomaron las primeras medidas preventivas. Entre los estudiantes que nunca habían consumido drogas, el $6 \%$ de los chicos y el $7 \%$ de las chicas respondieron que les gustaría probarlas (Andersson \& Hibell, 1996). Según los propios jóvenes, el acceso a las drogas se ha hecho mucho más fácil en los últimos diez años. Entre los chicos, el porcentaje que asegura haber tenido la oportunidad de probar alguna droga ha pasado del $11 \%$ de 1987 al $25 \%$ de 1996; entre las chicas, del
$11 \%$ al 20\% (Andersson \& Hibell, 1996). En resumen, puede afirmarse que existe una clara asociación en el tiempo entre la accesibilidad y la demanda entre los jóvenes (medida en función de la oportunidad y el deseo de consumir), así como con las experiencias de éstos en el consumo. Sin embargo, el vínculo causal entre los tres factores continúa sin estar bien definido.

Los conceptos de "accesibilidad" y "demanda" aparecen con frecuencia en las discusiones sobre la práctica de la prevención de drogas, aunque no parece existir una definición clara y sin ambigüedades de dichos términos. La mayoría de los investigadores parecen contentarse con señalar que tanto la accesibilidad como la demanda son conceptos de una gran complejidad que implican otros muchos factores. Einstein (1981), por ejemplo, establece una distinción entre la accesibilidad física, social, económica y legal. Smart (1977) subraya el hecho de que los estudios sobre las actitudes y las experiencias de los jóvenes ante las drogas sólo sirven para mostrar su forma de percibir dicha accesibilidad. No obstante, la accesibilidad real debe medirse de acuerdo con el número de lugares donde los jóvenes puedan comprar drogas o el número de personas que las vendan. El precio de las drogas y el tiempo que tarda el presunto comprador en encontrarlas serían otros indicativos de la accesibilidad real (Kleiman, 1992).

Basándose en numerosos estudios que han tratado de analizar la forma en que la accesibilidad afecta la situación actual de las drogas, Olsson (1995), llega a la siguiente conclusión: "Si el cannabis, por ejemplo, es fácil de conseguir, si el clima social es permisivo y una accesibilidad subjetiva incluye una inclinación a consumir o abusar de las drogas, serán muchos los individuos en situación de riesgo, aunque las tendencias individuales (por herencia, por las condiciones de vida en la infancia, por el entorno, por la personalidad) no sean las más adecuadas". Según Olsson, esto habla en contra de la liberalización de la política de las drogas, ya que aumentaría el número de consumidores y, consecuentemente, el número de personas que abusen de dichas sustancias. Para explicar la razón de que el consumo experimental de drogas se haya incrementado entre los jóvenes suecos, a pesar de la política restrictiva en dicho campo, Olsson (1995) habla de los recortes que "han afectado numerosas condiciones, repercutiendo directamente en la demanda". Pero Olsson no presenta la menor evidencia empírica que respalde su teoría.

Los trabajos que hayan contemplado al mismo tiempo la accesibilidad y la demanda son muy escasos. Esto queda en parte explicado por la dificultad que encierran ambos términos, especialmente la demanda. En un estudio sobre más de 4.600 alumnos, realizado en Ontario (Canada), Smart (1977) comprendió que la accesibilidad que percibían los jóvenes 
tenía un efecto significativo en aquellos estudiantes que afirmaban haber consumido drogas. Este efecto permanecía incluso teniendo presente el consumo de tabaco y alcohol. Su análisis no contenía ningún indicador sobre la demanda de drogas, y todas las variables eran medidas al mismo tiempo.

En un artículo posterior, Smart (1980) trabajó con la demanda y la accesibilidad. Esta última "haría también referencia a una serie de aspectos sociales, ya que las drogas son más fáciles de conseguir en unos grupos sociales que en otros. En determinados centros escolares, barrios o situaciones, los individuos que consumen drogas constituyen una mayoría". La demanda de dichas sustancias, que según Smart es el resultado de las circunstancias psicológicas o sociales, puede también variar entre diferentes colegios o áreas residenciales. En cuanto a la conexión entre estos dos factores, Smart (1980) afirma que no tiene por qué ser tan claro: "Allí donde la accesibilidad sea mucho mayor, el nivel de inclinación entre los consumidores puede ser menor que en otras situaciones de baja accesibilidad".

Los análisis efectuados por los centros escolares americanos tras la encuesta "The Monitoring the Future Study" han mostrado que las percepciones de los estudiantes sobre la accesibilidad no justifican las variaciones experimentadas en el consumo de drogas (Harrison \& Pottieger, 1996) sumo de drogas. En los últimos años, un número cada vez mayor de jóvenes ha manifestado su convencimiento de que, por ejemplo, fumar marihuana no es especialmente peligroso. Sin embargo, el hecho de que la accesibilidad no emerja como una explicación fundamental en este caso puede deberse a la edad relativamente elevada de los encuestados.

Desde 1991, los estudios realizados en los colegios americanos han incluido alumnos entre $8^{\circ}$ y $10^{\circ}$ (13-15 años). El incremento del consumo de drogas entre adolescentes de estas edades a lo largo de los noventa ha sido muy marcado. Entre los más jóvenes, el porcentaje de alumnos que afirma haber consumido cannabis ha aumentado desde el 6\% de 1991 al $18 \%$ de 1996. El incremento correspondiente entre los alumnos de $10^{\circ}$ ha pasado del $17 \%$ al $34 \%$. Las cifras que indican el acceso sin problemas a las drogas también han aumentado de forma considerable: del $42 \%$ de 1992 al 55\% de 1996 entre alumnos de $8^{\circ}$, y del $65 \%$ al $81 \%$ entre los alumnos de $10^{\circ}$ (The Monitoring the Future Study).

Los resultados obtenidos por todos los estudios indican, sin que ello sea ninguna sorpresa, que tanto la accesibilidad como el consumo aumentan con la edad. La conclusión a la que se ha llegado en Suecia es la misma. En un estudio realizado entre alumnos del $2^{\circ}$ año de secundaria en Upsala, el 18\% de los chi-

\section{tabla 1.- Experiencias con drogas ilegales entre alumnos del segundo año de la educación secundaria (tanto por ciento)}

\begin{tabular}{lcccccc} 
& \multicolumn{2}{c}{ Stockholm } & \multicolumn{2}{c}{ Botkyrka } & \multicolumn{2}{c}{ Täby } \\
& 1991 & 1996 & 1991 & 1996 & 1991 & 1996 \\
CHICOS & & & & & & \\
Deseaban consumirlas & 17 & 27 & 6 & 20 & 16 & 24 \\
Tuvieron oportunidad de hacerlo & 51 & 61 & 29 & 54 & 39 & 56 \\
Consumieron & 20 & 24 & 7 & 21 & 13 & 21 \\
CHICAS & & & & & & \\
Deseaban consumirlas & 10 & 20 & 8 & 16 & 6 & 24 \\
Tuvieron oportunidad de hacerlo & 40 & 52 & 30 & 44 & 34 & 48 \\
Consumieron & 9 & 14 & 8 & 9 & 6 & 17 \\
\hline
\end{tabular}

Más del $80 \%$ de los jóvenes americanos de 18 años de edad afirmaron tener acceso a las drogas. Esta cifra ha permanecido relativamente estable desde que comenzaron a tomarse medidas en 1975, aunque en los últimos años el porcentaje ha llegado a acercarse al 90\%. La accesibilidad, según dichos análisis, no es la causa principal, pero las cifras en descenso de los ochenta y el reciente aumento de los noventa podrían explicarse por la actitud de los jóvenes ante los riesgos para la salud que encierra el con- cos y el $16 \%$ de las chicas manifestaban su deseo de probar las drogas (Gymnasieelevers alkoholoch narkotikavanor, 1995). Esas cifras también incluían aquellos jóvenes que afirmaban haber consumido drogas en alguna ocasión. Casi el 30\% de los alumnos respondieron que a veces les habían ofrecido drogas. Cerca del $12 \%$ de los chicos y del $6 \%$ de las chicas de Upsala aseguraron haberlas probado.

CAN ha estudiado, asimismo, los hábitos relacionados con las drogas de los alumnos de las clases 
superiores de secundaria en Estocolmo y en los municipios de Botkyrka y Täby (ambos en la región de Estocolmo) en 1991 y 1996 (Grönberg, 1997). La tabla 1 muestra el porcentaje de chicos y de chicas que, en dichos centros escolares, han afirmado sentir deseos de probar las drogas, haber tenido ocasión de hacerlo o haberlas consumido.

Aproximadamente el doble de chicos que de chicas asegura haber consumido drogas en los cursos superiores de secundaria en la región de Estocolmo. Las diferencias entre ambos sexos son menores cuando se trata de hablar de sus deseos de consumir o de si se les ha presentado la oportunidad de hacerlo. El resultado más sorprendente de este estudio es la intensa variación observada en todas las variables entre los dos puntos de medición. Más de uno de cada dos estudiantes pertenecientes al área metropolitana de Estocolmo afirma haber tenido la oportunidad de probar alguna droga, y alrededor de uno de cada cinco chicos y una de cada diez chicas asegura haberlo hecho.

Es evidente que los jóvenes que manifiestan su interés en probar las drogas corren el riesgo de convertirse en un "nuevo" grupo de consumidores. Sin embargo, no todos los que muestran deseos de probar dichas sustancias tienen la oportunidad de hacerlo. En un estudio que comprendía todos los alumnos de $9^{\circ}$ y del tercer año de secundaria de Falkenberg (un pequeño municipio de 40.000 habitantes en la costa oeste de Suecia), alrededor del $5 \%$ de los jóvenes mostraban deseos de probar las drogas. Casi la mitad de este grupo señalaba que nunca se le había presentado la ocasión de hacerlo. Entre los alumnos de los cursos superiores de secundaria más del $8 \%$ indicaba que le hubiera gustado probar las drogas; de ellos, sólo dos de cada diez no habían tenido la oportunidad de hacerlo. En $9^{\circ}$, un $17 \%$ aseguraba haber tenido ocasión de probar las drogas, mientras que la cifra correspondiente entre los alumnos de los cursos superiores de secundaria era del 31\%. El porcentaje de los que habían consumido drogas en $9^{\circ}$ era el $7 \%$; en secundaria, el $12 \%$ (si se desea un informe más detallado de estos datos, ver Svensson, 1996ª 1996b).

Para ser breves, esta investigación pone en evidencia que la accesibilidad y la demanda están íntimamente ligadas entre sí, además de con la situación de los jóvenes ante la droga. Si, por ejemplo, fuera imposible conseguir drogas ilegales, aquellos que desean consumir alguna sustancia se volverían hacia el alcohol, "esnifarían" disolventes... o decidirían olvidarse del tema. Olsson (1995) sostiene que la accesibilidad de las sustancias ilegales también tiene una enorme influencia sobre el número de individuos que las consumen, y que si ésta es elevada origina por sí misma la demanda. El nivel de consumo de alcohol en cualquier país depende de forma crucial de su accesibili- dad. Se da por sentado que ocurre lo mismo con las drogas. A continuación, analizaremos cómo la accesibilidad y la demanda influyen en las actitudes y experiencias de los escolares ante la droga. Asimismo, nos preguntamos hasta qué punto una educación preventiva puede frenar los niveles de demanda y el consumo experimental de las drogas.

\section{MÉTODOY VARIABLES}

Durante el trimestre primaveral de 1996, la policía estuvo presente con su programa VÄGA en 40 centros escolares de Suecia (alumnos entre $7^{\circ}$ y $9^{\circ}$ ). Trece de ellos debieron tomar parte, antes de iniciar el trimestre, en una evaluación sobre la efectividad de dicho programa: la selección de los centros estuvo basada en la fecha en que debían empezar el programa y en la región donde se hallaban situados. Un centro escolar de control se emparejó con cada uno de los colegios VÄGA incluidos en la evaluación de tres variables: tamaño del centro (número de alumnos), región y grados medios. Alrededor de la mitad de los colegios estaban situados en Gothenburg y en las regiones de Bohus y Södermanland; el resto, en Malmöhus, Västmanland y Norrbotten.

Los datos se recogieron en unos cuestionarios que se pasaron en tres ocasiones: al comienzo y al fin del trimestre primaveral de 1996, y al terminar el mismo trimestre en 1997. Los alumnos de 13 y 14 años de edad respondieron de forma anónima durante las clases: se incluían preguntas sobre sus actitudes, conocimientos y experiencias con las drogas. En cuatro centros escolares (dos dentro del programa y dos de control) los últimos datos recogidos en la primavera del 97, sólo proporcionaron información de dos clases. Veintidós colegios (once dentro del programa y once de control), con un total de 2.000 alumnos, aportaron datos de interés después de pasar las tres mediciones. El número de cuestionarios completados y analizados ascendió a 1.680. El porcentaje de cuestionarios finales no contestados fue del $15 \%$. No se apreciaron diferencias entre los alumnos que habían tomado parte en el programa VÄGA y los que no habían participado en él, ni en sus preguntas no contestadas ni en sus factores ambientales (sexo, tipo de alojamiento, composición familiar, etc...).

\section{ANÁLISIS DE CONTEXTO: INFORMACIÓN DE LOS DIFERENTES NIVELES ANALIZADOS}

Para investigar en qué medida la demanda y la accesibilidad influyen en las actitudes y en las experiencias de los jóvenes con las drogas, y para determi- 
nar hasta qué punto el programa VÄGA infunde en éstos una actitud más negativa hacia las drogas, aplicamos en nuestro estudio el método conocido como análisis de contexto (Bryk y colaboradores, 1992; 1996). Este método de análisis estadístico nos permite examinar la interacción entre los factores de riesgo relacionados con el individuo, las características supuestamente asociadas con el resultado de la variable estudiada, así como las características del entorno social del individuo. Dicho método puede, asimismo, utilizarse para ver cómo la conexión entre los dos factores configura distintas situaciones o ambientes sociales. Ha sido puesto en práctica en campos muy diferentes, como por ejemplo en aquellas investigaciones de ciencia política interesadas en medir de qué forma las preferencias de un grupo de personas están influenciadas por las preferencias de sus compañeros de trabajo, aunque su utilización en el campo de la criminología es relativamente reciente. El análisis de contexto requiere información que pueda ser vinculada no sólo al individuo sino también al entorno o al grupo al que éste "pertenezca". La figura 1 ilustra un modelo de contexto simplificado. el factor de riesgo A en el nivel individual muestra lo que se conoce como el efecto de interacción. Ello significa que la combinación de esos dos factores de riesgo se considera relevante en el resultado final.

\section{VARIABLES INDIVIDUALES Y DE CONTEXTO}

La variable dependiente en nuestro estudio - actitudes y experiencias de los estudiantes con la droga consta de cuatro preguntas:

1) ¿Has consumido alguna vez en este trimestre hashish $u$ otros narcoticos? (respuestas establecidas: no; sí, una vez; sí, entre 2 y 5 veces; sí, más de 5 veces).

2) ¿Has deseado en alguna ocasión probar los narcóticos? (no; no lo sé; sí).

3) ¿Está bien probar los narcóticos para saber qué se siente? (no estoy de acuerdo; hasta cierto punto sí; estoy completamente de acuerdo).

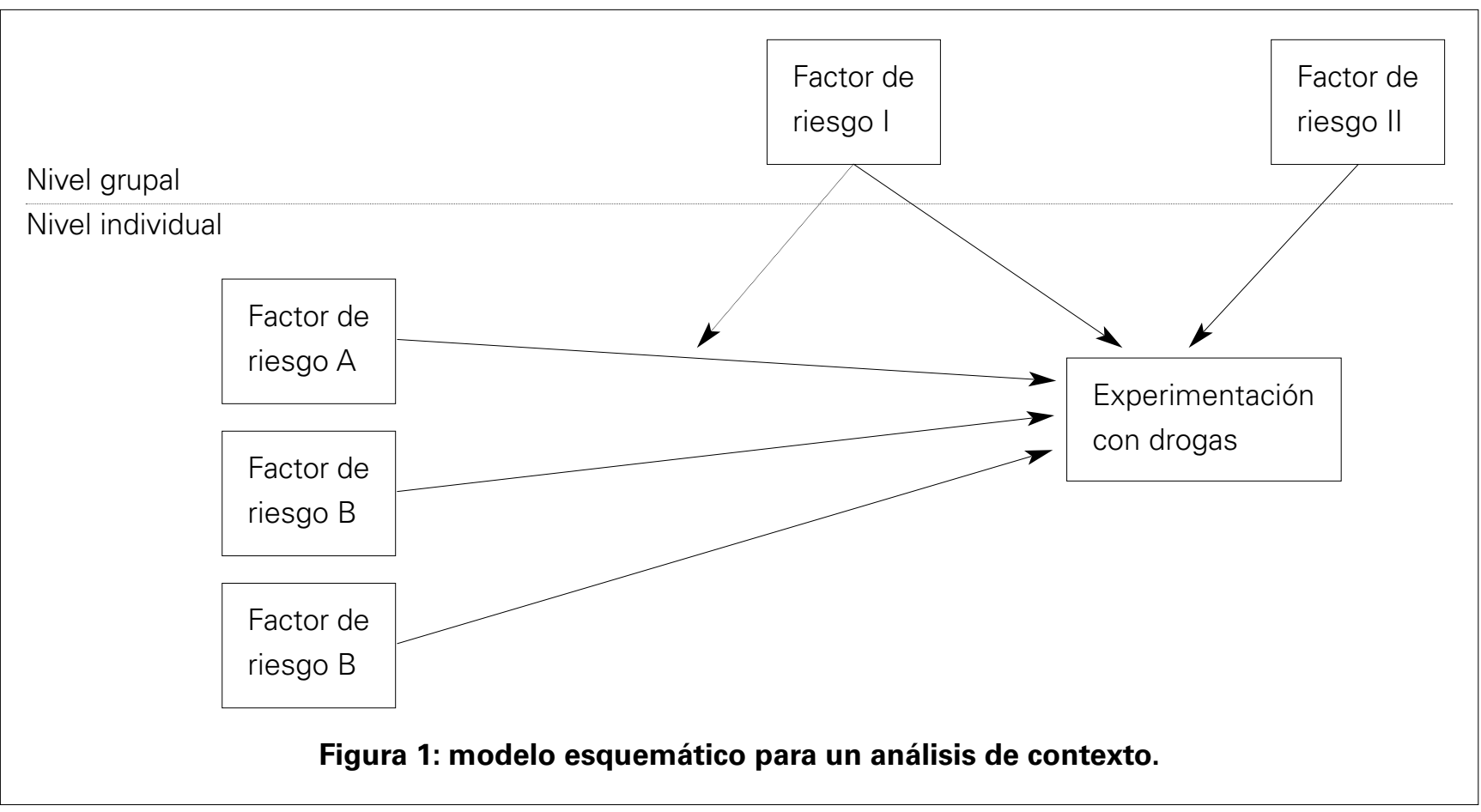

El modelo descrito en la figura 1 indica que las experiencias individuales con la droga están influenciadas por una serie de factores de riesgo de carácter individual $(\mathrm{A}-\mathrm{C})$. Dos variables de contexto (| y II) hacen referencia al entorno social (ej. compañeros de colegio o de clase) al que el individuo pertenece. De acuerdo con este modelo dichos factores de riesgo repercutirán también en las experiencias de ese individuo con las drogas. La flecha discontinua que va desde el factor de riesgo I en el nivel de grupo hasta
4) Si un amigo te ofreciera hashish u otro narcótico, ¿qué le responderías? (rotundamente no; probablemente no; no lo sé; probablemente sí; rotundamente sí).

Esta información se obtiene de las respuestas de los alumnos de $8^{\circ}$. Todas estas preguntas individuales muestran una lógica correlación entre sí, y el coeficiente de fiabilidad es de 0,78.

Las investigaciones realizadas tanto en Suecia como en otros países han identificado en el nivel indi- 
vidual un extenso número de factores de riesgo para el consumo experimental de las drogas. Los tradicionales factores de riesgo sociológico incluyen unas relaciones pobres del joven con sus padres, la falta de interés por los estudios y la compañía de amigos criminales (Sarnecki, 1985; Solarz, 1990; Andersson, 1993), A menudo se incluyen medidas para este tipo de variables en los estudios basados en cuestionarios, pero son numerosos los casos en que estos factores de riesgo también existen en estudios longitudinales y de base registrada (Torstensson, 1987; Kandel y colaboradores, 1986; Hawkins y colaboradores, 1995).

El análisis realizado a continuación incluye las siguientes variables individuales estudiadas en $8^{\circ}$ curso: sexo, tipo de alojamiento (bloque de apartamentos, etc...), estructura familiar (vive con uno de sus progenitores o con ambos), país de nacimiento (Suecia u otro lugar). Estos cuatro puntos se incluyen como variables de control. El 49\% de los encuestados eran chicas. Aproximadamente un tercio de los alumnos vivía en bloques de apartamentos, y la misma proporción lo hacía sólo con uno de sus progenitores. El $10 \%$ había nacido fuera de Suecia.

Se incluyeron tres variables para medir la relación entre los jóvenes y sus padres:

1) Cuando sales por la noche con tus amigos, ¿saben normalmente tus padres con quién estás? (respuestas establecidas: sí, siempre o casi siempre; sí, a veces; no, en raras ocasiones o nunca).

2) ¿Conocen tus padres a los padres de tus amigos? (cinco respuestas establecidas desde mis padres conocen a todos los padres de mis amigos hasta mis padres no conocen a ninguno de los padres de mis amigos).

3) ¿Con cuánta frecuencia haces planes divertidos con tu padre o con tu madre? (cuatro respuestas establecidas desde varias veces por semana hasta nunca o casi nunca). Estas preguntas también tienen una correlación entre sí y se combinan en un índice. Cuánto más elevado sea el valor en esta escala, más pobre es la relación con la familia.

Tres puntos relacionados con la ausencia sin permiso, el hacer o no los deberes y los resultados escolares, estrechamente vinculados entre sí, también han sido combinados. Existen dos puntos en el cuestionario sobre el tiempo con los amigos: ¿Con cuánta frecuencia sales con tus amigos los viernes y los sábados por la noche? Y ¿alguno de tus mejores amigos ha sido arrestado por la policía? Alrededor del $16 \%$ de los alumnos respondió que al menos dos de sus amigos habían sido detenidos por la policía, casi el $60 \%$ afirmó no tener ningún amigo que hubiera sido arrestado.

Por último, tres variables de contexto se incluyeron en el análisis: el porcentaje de alumnos que asegura- ban haber deseado probar los narcóticos, el porcentaje de los que señalaban haber tenido la oportunidad de hacerlo, y si el centro escolar al que pertenecían había participado o no en el programa VÄGA. Estos datos se obtuvieron a partir de mediciones efectuadas mientras los alumnos cursaban $7^{\circ}$. La confirmación de que la accesibilidad y la demanda deben contemplarse como factores de riesgo nos viene dada por un extenso estudio sobre las investigaciones publicadas: "Los factores de contexto que predicen el riesgo de abuso incluyen la accesibilidad y el precio del alcohol y de otras drogas, así como el hecho de que la sociedad sea tan permisiva ante el consumo del alcohol y de otras drogas" (Hawkins y colaboradores, 1995, 386). El programa $\vee \ddot{A G A}$ y otras campañas informativas tienen como objetivo funcionar como un elemento protector de contexto.

La proporción de alumnos de $7^{\circ}$ que en cada uno de los centros escolares indica haber deseado probar los narcóticos varía desde el 0 al 12\% (media 4,3 mediana 3,8\%). Las cifras correspondientes a los alumnos de $7^{\circ}$ que han tenido la oportunidad de probarlos oscila entre el 0 y el 13\% (media 6,4 - mediana $6,3 \%)$. Cuando todos los centros escolares se incluyen en el análisis, sólo queda una ligera correlación entre las valoraciones de los estudiantes sobre la accesibilidad y la demanda ( $r=.26, p=.124, n=22)$. Ello quiere decir, entre otras cosas, que los efectos de estas dos variables pueden evaluarse de forma simultánea.

\section{RESULTADOS}

Al finalizar el trimestre primaveral de 1996, el 3,7\% de los alumnos afirmaron haber deseado probar las drogas pero no haber tenido la ocasión de hacerlo. Un porcentaje algo más elevado $(5,7 \%)$ aseguró haber tenido la oportunidad pero no haber sentido interés por probar dichas sustancias. Sólo un $1,5 \%$ de los encuestados señaló haber deseado y haber tenido la ocasión de probar las drogas, pero no haberlo hecho. Un año después, cuando los alumnos respondieron a las mismas preguntas, el porcentaje de los que afirmaron sentir curiosidad e interés, pero no haber tenido la oportunidad de probar los narcóticos había aumentado en cierta medida $(4,3 \%)$, mientras que el porcentaje de los que sí habían tenido la oportunidad pero no habían sentido el menor interés por probarlos se duplicaba (11,7\%). Asimismo, el porcentaje de los alumnos que respondían que habían deseado y habían tenido la oportunidad de probar las drogas, se duplicaba, pasando del $1,5 \%$ al 3,3\%. El porcentaje que señalaba haber consumido alguna droga pasaba del $2,3 \%$ de $7^{\circ}$ al $4 \%$ de $8^{\circ}$. La tabla 2 muestra esas cifras, separando ambos sexos. 


\section{Tabla 2. Alumnos que afirman haber deseado, haber tenido la oportunidad}

y haber consumido drogas ilegales (tanto por ciento)

\begin{tabular}{lcccc} 
& \multicolumn{2}{c}{ CHICOS } & \multicolumn{2}{c}{ CHICAS } \\
& grado 7 & grado 8 & grado 7 & grado 8 \\
& & & & \\
Deseaban consumirlas & 3,7 & 4,7 & 3,7 & 4,0 \\
Tuvieron oportunidad de hacerlo & 7,6 & 12,4 & 3,9 & 10,9 \\
Deseaban consumirlas y tuvieron oportunidad de hacerlo & 2,0 & 3,4 & 1,0 & 3,3 \\
Consumieron & 3,6 & 5,7 & 1,0 & 2,3 \\
Número $(\mathrm{n})$ & 787 & 853 & 811 & 828 \\
\hline
\end{tabular}

No existen grandes diferencias entre los chicos y las chicas de $8^{\circ}$ en las preguntas sobre el interés en probar las drogas o las oportunidades de hacerlo. Sin embargo, los chicos que afirman haber consumido alguna droga doblan en número a las chicas. Dentro de los límites de este estudio es imposible saber hasta qué punto los chicos están exagerando sus experiencias. Son numerosos los estudios realizados con anterioridad en los que apenas se han encontrado diferencias entre los chicos y las chicas que afirmaban haber consumido narcóticos (ver Dolmén \& Lindström, 1991; Andersson \& Hibell, 1996). Sin embargo, otras investigaciones suecas ponen en evidencia que es mayor el número de chicos acusados de haber cometido delitos relacionados con las drogas o que sean conocidos por las autoridades debido a su consumo abusivo (Torstensson, 1987; Kühlhorn y colaboradores, 1996). La encuesta escolar más importante realizada en Estados Unidos, anteriormente mencionada en nuestro estudio (The Monitoring the Future Study, 1997), muestra que es mayor el número de chicos que consumen drogas, tanto en $8^{\circ}$ y $10^{\circ}$ como en $12^{\circ}$. Penning y Barnes (1982) llegan a la conclusión, después de revisar los estudios publicados, de que el consumo experimental de marihuana es más elevado entre los chicos. estudiantes con las drogas varían de unos centros escolares a otros, y, de ser así, en qué medida pueden "explicarse" esas diferencias con las variables de contexto. Si no hay diferencias entre los centros escolares en relación al consumo de drogas, ello significa que los factores de contexto pueden ser directamente excluidos y debemos analizar tan sólo las variables a nivel individual.

Un análisis de la cuestión nos demuestra que las actitudes y experiencias de los alumnos de $8^{\circ}$ con las drogas varían significativamente de unos centros escolares a otros (F-value $=2.499, p=.000)$. Aproximadamente un $3 \%$ de la varianza en la variable dependiente puede ser atribuida al nivel de contexto. La tabla 3 ilustra los efectos de la accesibilidad, de la demanda y del programa VÄGA sobre las actitudes de los estudiantes hacia las drogas y sus experiencias con las mismas.

Un primer análisis muestra que el número de alumnos que en $7^{\circ}$ afirmaban que les hubiera gustado probar los narcóticos y que habían tenido la oportunidad de hacerlo (tomando en consideración las respuestas de ambos sexos) tenía un efecto fácilmente apreciable sobre las experiencias de esos jóvenes con las drogas un año después. Es imposible determinar

Tabla 3. Efectos de la accesibilidad, de la demanda y haber seguido el programa VÄGA seguido en $7^{\circ}$ sobre las actitudes y las experiencias con las drogas ilegales en $8^{\circ}$. Análisis de la regresión jerárquica (HLM).

\begin{tabular}{lcccc} 
Variables & Coeficiente & Standard & T-ratio & p. \\
Demanda & 2,122 &, 705 & 3,008 &, 008 \\
Accesibilidad & 1,625 &, 584 & 2,784 &, 013 \\
VÅGA & $-0,21$ &, 036 &,- 568 &, 577 \\
\hline
\end{tabular}

\section{EFECTOS DEL CONTEXTO}

La primera cuestión que debemos plantearnos es hasta qué punto las actitudes y las experiencias de los cuál es el factor de riesgo -demanda o accesibilidadque tiene mayor importancia en ese año que ha transcurrido, puesto que los dos factores tienen aproximadamente la misma influencia sobre la variable 
dependiente (los coeficientes de regresión standard tanto para la demanda como para la accesibilidad son 0.08). Por otra parte, no se aprecia el menor efecto en las experiencias con las drogas de los alumnos de $8^{\circ}$ cuando éstos han participado en el programa VÄGA en $7^{\circ}$.

Cuando el análisis se efectúa separando los dos sexos, encontramos que dichos efectos sólo pueden aplicarse a los chicos. Entre las chicas, no puede afirmarse que ninguno de los factores de contexto tenga unos efectos estadísticamente verificables sobre sus experiencias con las drogas en $8^{\circ}$. La razón de ello es que las chicas, en general, tienden a desarrollar una actitud mucho más negativa hacia las drogas que los chicos; son muy pocas las que responden haber consumido narcóticos. Asimismo, el método de análisis empleado es incapaz de captar los posibles efectos de las variables independientes, por ejemplo el error standard es demasiado elevado.

Una evaluación anterior de los efectos a corto plazo del programa $\vee \ddot{A G A}$, en la que los alumnos de $7^{\circ}$ respondieron a dos cuestionarios al comenzar y al finalizar el trimestre primaveral, tampoco pudo demostrar que haber seguido dicho programa tuviera algún efecto apreciable sobre los hábitos de consumo de alcohol, narcóticos o tabaco de los estudiantes (Lindström, 1996). La figura 2 ilustra el fracaso del programa $\vee \ddot{A G A}$ a la hora de satisfacer las expectativas diferencia el programa educativo de prevención de drogas más empleado en los colegios americanos y, sin embargo, no hay duda de que también es el menos efectivo. El informe concluye diciendo que "los métodos de prevención de drogas que han mostrado ser más efectivos son los menos utilizados, mientras que los métodos que no han sabido probar su efectividad o no han sido aún evaluados adecuadamente son los que gozan de mayor difusión" (Silvia \& Thorne, 1997, E-23).

En una investigación encargada por el Congreso de los Estados Unidos, Sherman (1997) observa que "al decidir apoyar el programa DARE, el Congreso ha pasado por alto la existencia de otros programas de prevención escolar cuya efectividad sí estaba científicamente probada y era mucho mayor que la del DARE. Es un hecho cierto que la versión más extendida del DARE no tiene el menor efecto a la hora de prevenir el consumo abusivo de drogas". Incluso a pesar de no existir una evidencia empírica que apoye el programa, el gobierno americano (en su "Estrategia Nacional para el Control de Drogas: 1997") describe el programa DARE como un programa de prevención de drogas de gran éxito. Cerca de 25.000 oficiales de policía intervienen en dicho programa en Estados Unidos, lo que según algunos cálculos supone un coste de más de 700 millones de dólares al año (Clayton y colaboradores, 1996).

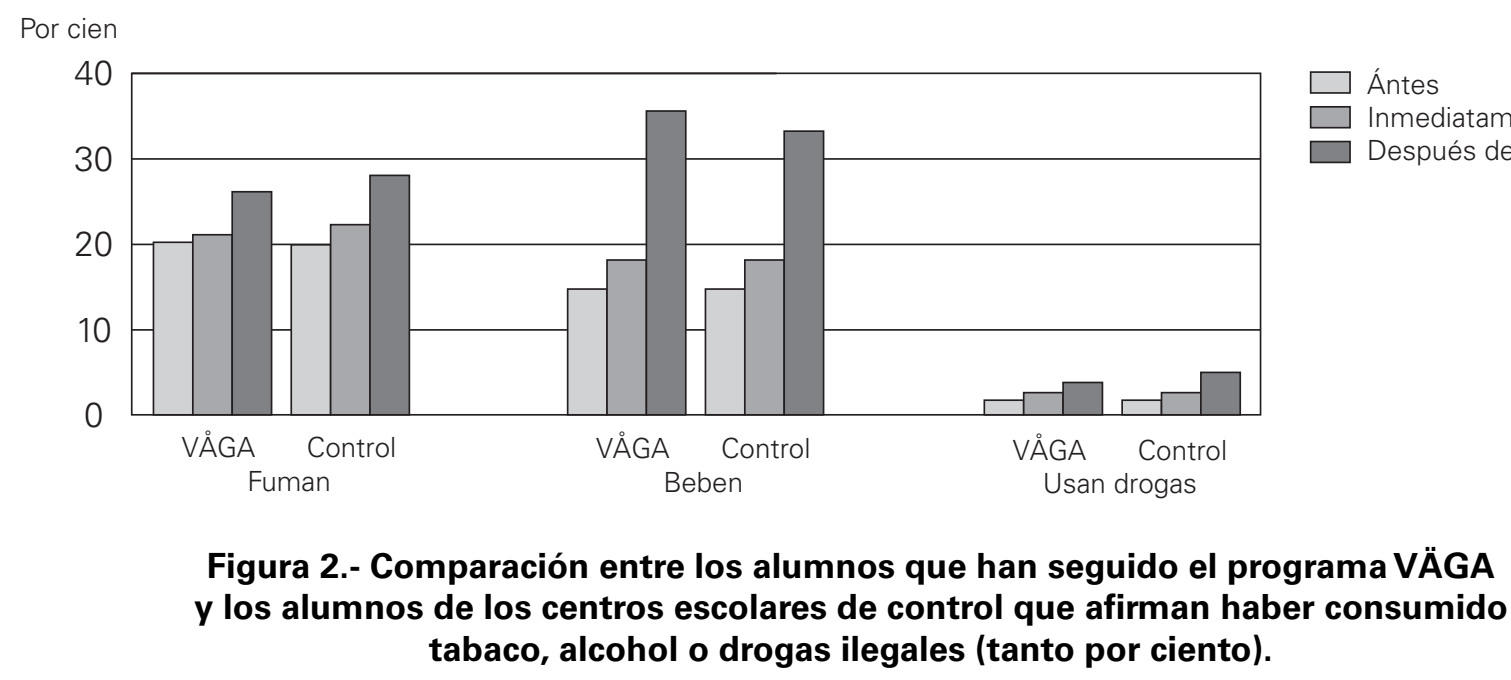

relacionadas con la prevención del consumo de drogas entre los jóvenes un año después de haber seguido el programa.

Los resultados de esta evaluación sueca sobre el programa VÄGA están en línea con los obtenidos en las evaluaciones americanas sobre el programa DARE. A petición del Departamento Americano de Educación, Silvia y Thorne (1997) realizaron un estudio sobre los programas de prevención de drogas de más amplia difusión y evaluaron sus efectos. DARE es con
La primera conclusión preliminar que puede extraerse de nuestro análisis es que las actitudes y las experiencias con las drogas en $8^{\circ}$ se ven influenciadas por el porcentaje de alumnos que aseguran haber tenido la oportunidad y haber deseado probar las drogas en $7^{\circ}$. El efecto de estos dos factores es aproximadamente el mismo. Por el contrario, el hecho de haber participado en un programa VÄGA no tiene el menor efecto en las actitudes que presentan los jóvenes hacia las drogas ni en sus experiencias con las 
mismas. Una importante pregunta que debemos plantearnos es hasta qué punto los efectos de los factores agregados - accesibilidad y demanda - se mantienen al tomarse en consideración las características individuales (la relación con los padres, interés por el colegio, salidas con los amigos...). Y ésa es la cuestión que pasamos a analizar en el siguiente punto.

\section{EFECTOS DE LOS FACTORES DE RIESGO INDIVI- DUALESY DE CONTEXTO}

Hasta el momento no hemos prestado atención a los posibles factores de riesgo a nivel individual, es decir aquellas características que suponemos incrementan la probabilidad de que los alumnos adopten una actitud más positiva ante las drogas. En este análisis final tomamos en cuenta tanto los factores de riesgo individuales como de contexto, así como tres efectos de interacción. El primer efecto de interacción examina hasta qué punto el programa VÄGA tiene algún impacto en la asociación entre el efecto de los compañeros y las experiencias individuales con las drogas. Los otros dos se preocupan de los efectos de la demanda y de la accesibilidad a nivel escolar y de la influencia de los compañeros a nivel individual. Dado que las variables agregadas no poseen un efecto que pueda ser apreciado en las actitudes de las chicas hacia las drogas, el análisis se ocupa únicamente de los chicos. La tabla 4 nos muestra los resultados del análisis de contexto.
Todos los factores de riesgo individuales: relación pobre con los padres, bajo interés escolar e influencia de los compañeros muestran un efecto estadísticamente significativo sobre la variable dependiente. Por el contrario, ninguna de las variables de control muestra un efecto significativo. Tal como hemos visto con anterioridad, participar en un programa VÄGA no tiene el menor efecto sobre la variable dependiente, como tampoco la combinación de haber participado en dicho programa y la influencia de los compañeros tienen algún efecto que pueda ser medido sobre las experiencias de los jóvenes con las drogas. La asociación entre la influencia de los compañeros y las actitudes hacia las drogas tiene la misma intensidad entre los que han seguido el programa que entre los que no lo han hecho ( $r=.35, p=.000)$. En la medida en que no existen efectos apreciables que justifiquen una inversión tan considerable como la del programa $\vee \ddot{A} G A$, es lógico cuestionar la conveniencia de que la policía continúe gastando una parte tan importante de su presupuesto en dicho programa.

De las dos variables que miden la demanda y la accesibilidad en $7^{\circ}$, sólo la segunda tiene un efecto significativo. En los centros escolares donde los alumnos reconocen una mayor accesibilidad - siendo iguales las demás características -, hay un número mayor de jóvenes que presentan una actitud menos negativa hacia las drogas. Ninguno de los efectos de interacción resultaron tener el menor impacto sobre las actitudes de los alumnos hacia las drogas.

En evaluaciones anteriores del programa DARE, algunos estudios han señalado la posible existencia de

\section{Tabla 4.- Efectos de los factores de riesgo individuales y de contexto en las experiencias con las drogas de los alumnos varones. Análisis de la regresión jerárquica (HLM).}

Variables

Nivel individual

Acomodación

Orígenes nacionales

Composición familiar

Interesado en la escuela

Contacto con los padres

Influencia de los pares

Nivel escolar

VÅGA

DEMANDA

ACCESIBILIDAD

Interacción

VÅGA e influencia de los pares

DEMANDA e influencia de los pares

ACCESIBILIDAD e influencia de los pares
Coeficiente

Standard

T-ratio $\mathrm{p}$

0,007

0,036

0,028

0,081

0,022

0,142

0,050

0,137

0,893

0,081

0,048

0,448

0,659

0,012

0,575

0,572

0,010

6,971

0,000

0,023

2,267

0,036

6,107

0,000
0,001

1,288

2,098

0,031

1,155

1,095
0,058

1,081

0,945

0,048

0,877

0,780
0,141

1,192

0,890

0,249

2,221

0,039

0,531

0,639

0,204

1,318

0,177 
efectos de interacción. Rosenbaum y sus colaboradores (1994), por ejemplo, hallaron que el consumo de alcohol era menor entre las chicas que habían seguido el programa que entre las chicas del grupo de control. Entre los chicos sucedía todo lo contrario: los que reducían el consumo de alcohol eran más numerosos dentro del grupo de control que dentro del grupo que había seguido el programa DARE. Clayton y sus colaboradores (1991) encontraron en su estudio que el consumo de drogas entre los chicos que presentaban una elevada puntuación en el factor que mide la búsqueda de sensaciones era menor después de su participación en el programa DARE que entre los chicos del grupo de control. Sin embargo, ambos estudios hacen hincapié en que los efectos de esta interacción deben ser interpretados con enorme cautela. Es de suma importancia identificar de forma empírica grupos específicos que puedan beneficiarse del programa, aunque cualquier efecto positivo debe ser comprobado nuevamente antes de sacar conclusiones en firme. Asimismo, es importante tratar de identificar aquellos grupos que pudieran ser influidos negativamente por el programa.

El análisis de los efectos de interacción en nuestra evaluación del programa $\vee \ddot{A} G A$ en Suecia reveló que un $18 \%$ de los alumnos de $8^{\circ}$ que afirmaban no estar interesados por el trabajo escolar y que habían seguido dicho programa deseaban probar las drogas. El porcentaje correspondiente a los alumnos que no habían seguido el VÄGA era del $11 \%$. En los datos recogidos antes de comenzar el mencionado programa, un $8 \%$ de los jóvenes que mostraban falta de interés hacia el trabajo escolar, tanto en los colegios que seguían el VÄGA como en los de control, aseguraban haber querido probar las drogas. Es obvio que esta clase de efecto se opone a las intenciones del programa. A pesar de ello, el informe sobre la prevención del alcohol y de las drogas publicado por el Instituto Nacional de Salud Pública en 1995 nos recuerda que ésta es una posibilidad que debe tenerse en cuenta:

El mensaje debe ser simple, claro y, fundamentalmente, adaptarse al grupo que constituye nuestro objetivo. Una información de suma importancia para un grupo determinado, puede tener el efecto contrario en otro grupo. Un ejemplo de ello es la información sobre las drogas: qué clase de drogas hay, qué aspecto presentan, qué precio tienen, cuáles son sus efectos... Esta clase de información es importante para los padres de adolescentes; sin embargo, si va destinada a estos últimos puede servir para educarles en el consumo y aumentar su interés (Folkhälsoinstitutet, 1995).

\section{CONCLUSIONESY DISCUCIÓN}

En Suecia se ha debatido ampliamente cuál sería la política más efectiva frente a la droga, o los resultados perseguidos por unas leyes restrictivas y represivas. Kühlhorn y sus colaboradores (1996) sostienen que los pasos dados con el fin de limitar y controlar el tráfico de drogas en la calle (endureciendo las penas por vender o comprar drogas, aumentando el número de policías dedicados a luchar contra su tráfico) lograrán reducir el número de jóvenes que prueben las drogas. Sin embargo, otros investigadores insisten en que ni las grandes brigadas antidroga ni unos castigos más duros para los delitos relacionados con el tráfico y el consumo de drogas reducirán el número de consumidores, y un control excesivo tendrá efectos negativos, como por ejemplo la indiferencia a los principios legales (Tham, 1996). Las políticas seguidas en el campo de la droga no se deciden simplemente tomando en consideración la evidencia científica de esta u otra línea política. Fundamentalmente, la elección entre una política restrictiva o una estrategia de liberalización, descriminalización o legalización se realiza en base a una serie de consideraciones políticas. En nuestra situación actual, no existe la menor evidencia de que la política seguida en el campo de las drogas en Suecia vaya a ser nuevamente analizada o revisada en un futuro previsible.

Los esfuerzos realizados en distintos campos para influenciar en las actitudes de los jóvenes hacia las drogas, así como en su consumo de las mismas, son muy numerosos. Siempre se ha pensado que limitar la accesibilidad es tarea de la policía y de las autoridades aduaneras, mientras que mantener controlada la demanda proporcionando la información adecuada es responsabilidad de las instituciones educativas. El sistema escolar, por ejemplo, juega un importante papel al proporcionar datos de relevancia sobre los efectos nocivos de las drogas. Sin embargo, apenas se han realizado investigaciones de forma sistemática para establecer el verdadero impacto de esa información y para determinar quién es el más cualificado para darla. Sería lógico pensar que si a los jóvenes se les habla de las consecuencias negativas del consumo abusivo de drogas, éstos optarían por rechazarlas. Desgraciadamente, sin embargo, los resultados de nuestro estudio muestran que las cosas no son tan sencillas. Existen, por supuesto, innumerables razones por las que los jóvenes desean experimentar, pero una de ellas es el hecho de que las drogas están allí mismo, al alcance de su mano.

La participación cada vez mayor de la policía en el terreno de la prevención de drogas en general y en el programa educativo VÄGA en particular no puede ser justificada si consideramos el presupuesto invertido o los resultados obtenidos. El papel de la policía - si se decide en primer lugar que el esfuerzo VÄGA merece la pena - podría ser realizado por otras personas. Los fondos policiales deberían dedicarse a otras áreas donde resultaran más beneficiosos y efectivos. El análisis de los mercados locales de la droga (en qué zonas 
se vende, quién lo hace, cuándo y dónde, etc...), así como la colaboración con las compañías que ofrecen alojamiento, con los comerciantes y con los servicios de asistencia social, han proporcionado buenos resultados en otros países, al menos a corto plazo (Weisburd \& Green, 1995). Esta clase de estrategia orientada a cada localidad se basa en la siguiente premisa: “Las oportunidades para el tráfico de drogas pueden reducirse poniendo en el punto de mira las situaciones y los lugares que facilitan el consumo de drogas antes que las personas que consumen o venden dichas sustancias" (Green, 1995, 737). Y ésas son las tareas que deberían encomendarse a la policía local.

Es evidente que los centros escolares continuarán trabajando en el campo de la prevención de drogas. Sin embargo, sería lógico que se diera prioridad al alcohol sobre las drogas ilegales y que los colegios, colaborando más estrechamente con otras instituciones de la sociedad, encabezaran este esfuerzo. La policía debería dar a los alumnos la oportunidad de conocer a las víctimas de un delito, a los familiares de los toxicómanos, a asistentes sociales, a trabajadores relacionados con la libertad condicional de algunos presos, así como de visitar autoridades que persigan delitos relacionados con las drogas y tribunales de justicia. Asimismo, las charlas con antiguos toxicómanos e infractores de la ley, que actualmente se celebran con bastante frecuencia en los colegios, pueden ayudar a una mejor comprensión del problema por parte de los alumnos. En cualquier caso, no existe la menor evidencia de que este tipo de programa resulte más efectivo si se pone en práctica en cursos anteriores, a pesar de que el profesor encargado de cada clase debe empezar a trabajar el tema. Puesto que un gran número de alumnos tendrán sus primeras experiencias con el alcohol y el tabaco, y muy pocos con las drogas ilegales, es ahí donde la educación debería empezar. El programa de prevención debería continuarse en los últimos cursos de secundaria.

Noruega inició un importante programa de prevención en sus colegios en otoño de 1997. En las clases de sociales, historia y religión los alumnos tienen la oportunidad de hablar con sus profesores e invitar a conferenciantes que disertan sobre temas éticos, abuso de drogas y delitos relacionados con el tráfico y el consumo de dichas sustancias. Deberíamos ser capaces de aprender del éxito de este programa.

\section{BIBLIOGRAFÍA.}

Andersson, B. R Hibell, B. (1995): Skolelevers dOrogvanor 1995 [Schoolchildren's drug habits 1995]. Centralforbundet for alkohol- och narkoti-kaupplysning, Stockholm: CAN
Andersson, B. 8c Hibell, B. (1996): Skolelevers drogvanor 1996 [Schoolchildren's drug habits 1996]. Stencil-serie nr 31. Centralforbundet for al-kohol- och narkotikaupplysning, Stockholm: CAN

Andersson, C. (1993): The Children of Marie: Adolescent Substance Abusers, their Families and Schooling. Uppsala universitet

Botvin, G.J. (1996): Substance Abuse Prevention Through Life Skills Training. In: Peters, R. DeV. R McMahon, R.J. (eds.): Preventing Childhood Disorders, Substance Abuse, and Delinquency. Thousand Oaks: SAGE

Bryk, A.S. 5c Raudenbush, S.W. (1992): Hierarchical Linear Models: Application and Data Analysis Methods. Newbury Park: SAGE

Bryk, A.S. R Raudenbush, S.W. 5c Congdon, R. (1996): Hierarchical Linear and Non-linear Modelling with the HLM/2L and HLM/3L Programs. Chicago: Scientific Software International

Clayton, R.R. R Cattarello, A. 8c Walden, ICP. (1991): Sensation Seeking as a Potential Mediating Variable for School-Based Prevention Intervention: A Two-Year Follow-Up of DARE. Health Communication 3: 229-239

Clayton, R.R. 8c Leukefeld, C.G. 5c Harrington, N.G. R Cattarello, A. (1996): DARE (Drug Abuse Resistance Education): Very Popular but Not Very EfFective. In: McCoy, C.B. 8c Metsch, L.R. R Inciardi, J.a. (eds.): Intervening With Drug-Involved Youth. Thousand Oaks: SAGE

Davis, R.C. 8c Lurigio, A.J. (1996): Fighting Back: Neighborhood Antidrug Strategies. Thousand Oaks: SAGE

Dolmen, L. R Lindstrom, P. (1991): Skola, livsstil och brott [School, way of life and crime]. BRA-rapport 1991:3 Brattsforebyggande radet. Stockholm: Allmanna forlaget

Einstein, S. (1981): Drug Use Intervention Concepts: Availability. The International Journal of the Addictions 16 (3), iii-iv

Folkhalsoinstitutet [National Institute of Public Health] (1995): Nationell handlingsplan for alko-hol- och drogforebyggande arbete [National action plan for alcohol and drug prevention]. FHI 1995:50. Stockholm

Gottfredson, D.C. (1997): School-Based Crime Prevention. (Chapter 5). In: Sherman, L.W. R Gottfredson, D. 8c MacKenzie, D. $R$ Eck, J. $R$ Reuter, P. 8c Bushway S. (eds.): Preventing Crime: What Works, What Doesn't, What's Promising'. A Report to the United States Congress, Prepared for the National Institute of Justice. Department of Criminology and Criminal Justice, University of Maryland

Green, L. (1995): Cleaning Up Drug Hot Spots In Oakland, California: The Displacement and Diffusion EfFects. Justice Quarterly 12 (4): 737-754

Green, L. (1996): Policing Places with Drug Problems. Sage

Gronberg, K. (1997): Gymnasieelevers drog-vanor 1991 och 1996 [Upper secondary school students' drug habits in 1991 and 1996]. Central-forbundet for alkohol- och narkotikaupplysning, Stockholm: CAN

Gymnasieelevers alkohol- och narkotikavanor 1995 [Upper secondary school students' alcohol and drug habits in 
1995]. Folkhalsoinstitutet i samarbete med Uppsala kommun

Harrison, L.D. 8c Pottieger, A.E. (1996): The Epidemiology of Drug Use Among American Youth. In: McCoy, C.B. R Metsch, L.R. 5c Inciar-di, J.a. (eds.): Intervening with Drug-Involved Youth. Thousand Oaks: SAGE

Hawkins, J.d. 8c Arthur, M.W. Bc Catalano, R F. (1995): Preventing Substance Abuse. In: Tonry, M. R Farrington, D.P. (eds.): Building a Safer Society. Crime and Justice, Vol. 19. Chicago: University of Chicago Press

Kandel, D.B. Bc Simcha-Fagan, O. 8c Davies, M. (1986): Risk Factors For Delinquency and Illicit Drug Use from Adolescence to Young Adulthood. Journal of Drug Issues 16: $67-90$

Kiihlhorn, E. 8c Kassman, A. 8c Ramstedt, M. (1996): Atgarderna mot drogbrottsligheten: Ett naturligt experiment inom narkotikapreventionen [Measures against drugrelated crime: A natural experiment within drug prevention]. In: Ahlberg, J. (ed.): Brottsutvecklingen [Development of crime]. Brottsforebyggande radet

Kleiman, M.A.R. (1992): Against Excess. Drug Policy for Results. BasicBooks

Lag- och rattundervisning [Law and justice education] (1995) (handbook). Stockholm: Riks-polisstyrelsen

Lindstrom, P. (1996): En utvardering av VAGA-programmets korttidseffekter [Evaluation of the short-term effects of the VAGA programme]. Solna: Polishogskolan

Lindstrom, P. (1997): ANT-undervisning i Stockholms skolor [ANT education in Stockholm schools] (manuscript)

Lindstrom, P. R Pauloff', A. (1997): Hur efFek-tivt ar det amerikanska VAGA-programmet? [How effective is the American DARE programme?] Solna: Polishopkolan (manuscript)

The Monitoring the Future Study (1997): www.isr.umich.edu/src/mtf/index.html

Olsson, O. (1995): Liberalisering au narkotika-politiken [Liberalization of drug policy]. Stockholm: Folkhalsoinstitutet och Centralforbundet for alkohol- och narkotikaupplysning

Orlandi, M.O. (1996): Prevention Technologies for Drug-Involved Youth. In: McCoy, C.B. R Metsch, L.R. 5c Inciardi, J.a. (eds.): Intervening With Drug-Involved Youth. Thousand Oaks: SAGE

Penning, M. R Barnes, G.E. (1982): Adolescent Marijuana Use: A Review: International Journal of Addiction 17: 749-791

Rosenbaum, D.P. R Flewelling, R.L. 8c Bailey, S. 8c Ringwalt, C.L. RWilkinson, D.L. (1994): Cops in the Classroom: A Longitudinal Evaluation of Drug Abuse Resistance Education (DARE). Journal of Research in Crime and Delinquency 31 (1): 3-31
Sarnecki, J. (1985): Vem blir sprutnarkoman? [Who becomes an addicted intravenous user?]. Brottsforebyggande radet

Sherman, L.W. (1997): Conclusions: The Effectiveness of Local Crime Prevention Funding (Chapter 10). In: Sherman, L.W. R Gottfredson, D. 5c MacKenzie, D. R Eck, J. 5c Reuter, P. 5t Bushway, S. (eds.): Preventing Crime: What Works, What Doesn't, What's Promising'. A Report to the United States Congress, Prepared for the National Institute of Justice. Department of Criminology and Criminal Justice, University of Maryland

Silvia, E.S. RThorne, J. (1997): School-Based Drug Preytntion Programs: A Longitudinal Study in Selected School Districts. Executive Summary. North Carolina: Research Triangle Institute

Skretting, A. (1996): Ungdommer og rusmidler [Youths and intoxicants]. Rusmiddeldirektoratet

Smart, R G. (1977): Perceived Availability and the Use of Drugs. Bulletin on Narcotics 29 (4): 59-63

Smart, R.G. (1980): An Availability-Proneness Theory of Illicit Drug Abuse. Theories on Drug Abuse. Rockville, MD: National Institute on Drug Abuse

Solarz, A. (1990): Vem blir drogmissbrukare [Who becomes a drug abuser]. Bra-rapport 1990:3. Brottsforebyggande radet

Svensson, R. (1996a): Ungdomars narkotika-vanor i Falkenbergs kommun. En sjalvrapporte-rande totalundersokning bland clever i \&skurs nio [Youths' drug habits in Falkenberg municipality. A self-report study among students in grade nine]. Kriminologiska institutionen, Stockholms univer-sitet

Svensson, R. (1996b): Ungdomars narkotika-vanor pi Falkenbergs gymnasieskola. En sjalvrap-porterande totalundersokning bland clever i ars-kurs tre [Youths' drug habits in Falkenberg upper secondary school. A selfreport study among third-year upper secondary school students]. Barn- och utbildningsnamnden, Falkenbergs kommun

Tham, H. (1996): Den svenska narkotikapoliti-ken - en restriktiv och framgkngsrik modell? [Swedish drug policy a restrictive and successful model?] Nordisk Alkoholtidskrift 13 (4): 179-193

Torstensson, M. (1987): Drug-Abusers in a Metropolitan Cohort. Project Metropolitan Research Report No. 25. Department of Sociology, University of Stockholm

U.S. Department of Health and Human Services (1995): Annual Survey Shows Increases in Tobacco and Drug Use by Youth. Washington: HHS NEWS

Weisburd, D. R Green, L. (1995): Policing Drug Hot Spots: The Jersey City Drug Market Analysis Experiment. Justice Quarterly 12 (4): 711-736

VEGA: Lararhandledning [Guidelines for teachers in the VAGA programme] (1993). Riks-polisstyrelsen. 
